\title{
A wearable system to detect urine leakage based on a textile sensor.
}

\author{
Marc Martínez-Estrada, Raul Fernández-García, Ignacio Gil \\ Universitat Politècnica de Catalunya, Dept. Electronic Engineering, Terrassa, Spain \\ marc.martinez.estrada@upc.edu
}

\begin{abstract}
In this work, a wearable system to detect urine leakage based on a textile sensor is proposed. The system consists of a capacitive sensor embroidered with a conductive yarn in a cotton/polyester substrate. A microcontroller (MCU) with a wireless module for signal acquisition and a smartphone for monitoring complete the overall system. The capacitive sensor behaviour was tested in a climatic chamber between $30 \%$ and $80 \%$ of relative humidity (RH) with a LCR meter and charge/discharge method directly connected to the MCU. The results show that the proposed system is able to detect urine leakage even though when the patient wears a diaper. The functionally allows to develop a real time monitoring system which can help nursing and medical assistants to take care of elderly and disabled people and improve the quality of life of these patients.
\end{abstract}

Keywords—wearable; sensor; humidity; device; smart textile.

\section{INTRODUCTION}

Nowadays, the electronic and communication technology can help to improve the quality of life of population. Several wearable devices can be found in the literature and the market to analyze several biological fluids [1], gait analysis based on inertial measurement units [2], evaluation of patient with Parkinson's disease [3], the protection and monitoring of firefighter's [4], etc. However, in order to guarantee a full wearability and comfort of these devices a lot effort for integrating this type of sensing functionalities on textile materials has been developed [5]. Some textile sensors can be found in the literature for measuring limb motion [6], spinal extension [7], finger touch and pressure [8], humidity [9], etc.

The urine leakage and urinary incontinence is a disorder with high social and economic cost that can affect up to $33 \%$ of patients in health centers [10]. Despite of this problem can be solved by means of diapers, in some cases, the patient doesn't know if the urinary incontinence has been produced. Due to this fact, in hospitals and retirement homes, the nurses should check periodically the patients in order to know if the diaper should be replaced which implies an associate social and economic cost.

In order to increase the quality of life of these patients and also to reduce the workload of nursing and medical assistants of retirement homes and hospitals in this paper a wearable system to detect urine leakage and/or urinary incontinence is presented. The system is based on a capacitive textile moisture sensor previously published by the authors [9]. The proposed system is able to detect urine leakage also when the patients wears a diaper.

\section{MATERIALS AND METHODS}

Fig. 1 depicts the bloc diagram of the proposed wearable system. The urine leakage system consists of: an embroidered textile capacitive sensor to detect the leaks, a conventional electronic circuit, which is responsible for the acquisition and wireless transmission of the information provided by the sensor and finally, a smart phone to receive the information and notify if a urine leakage has been produced.
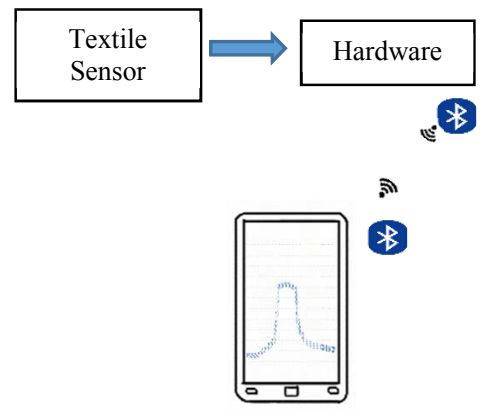

Fig. 1. Proposed bloc diagram

The urine leakage sensor is embroidered with a commercial Shieldex 117/17 dtex 2-ply conductive yarn with a polyester/cotton support yarn. Shieldex yarn is made by filaments of polyamide coated by pure silver, which provides a good conductivity and fastness to washing [11]. The sensor has been embroidered on a commercial cotton underwear due to both, its hydrophilic behaviour and the comfort that cotton provides to the user. The sensor has been located in the underwear area where higher humidity will be present. Fig. 2 shows the underwear with the embroidered sensor. 


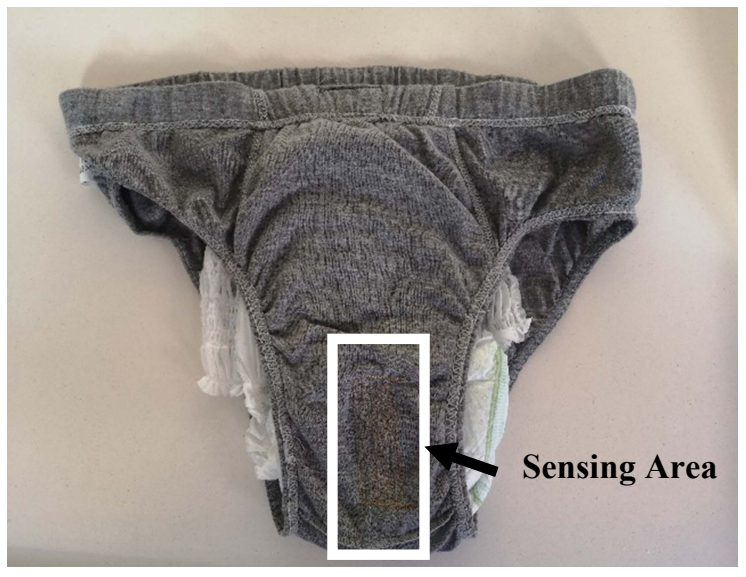

Fig. 2. Embroidered sensor in an underwear with a diaper

To embroider the pattern in the cotton substrate a Singer Futura XL-550 embroidery machine was used. The pattern was embroidered with a satin fill stitch structure looking for the best homogenous yarn distribution. The details and main sensor dimensions are shown in Fig. 3 .

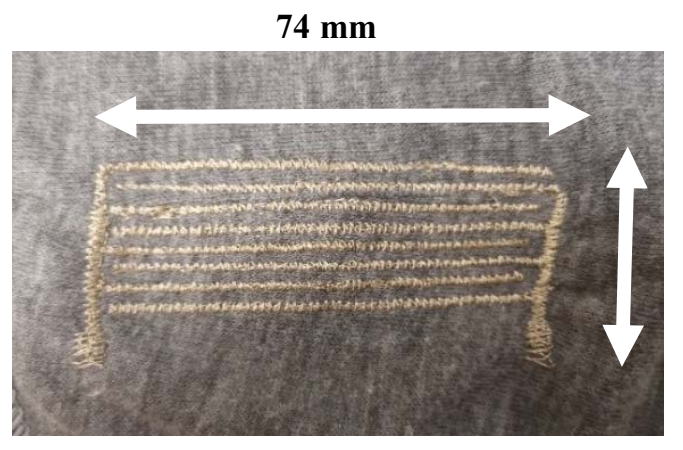

$32 \mathrm{~mm}$

Fig. 3. Layout and dimension detail of the proposed moisture sensor

In order to determine the capacitance sensor behavior, the textile sensor was tested in a $C C K-25 / 48$ Dycometal climatic chamber and the sensor capacitance was measured from $30 \%$ to $80 \%$ of relative humidity by means of an external Rohde \& Schwarz HM8118 LCR meter.

Once the sensor behavior was defined, the device was directly connected to MCU by means of an integrated analog to digital converter and the charge/discharge method was used to measure the capacitance of the sensor [12]. The MCU will be attached with snaps to the clothes, the width does not be bigger than a belt buckle. These capacitate values were directly wireless transmitted with a Bluetooth connection to a smartphone. The MCU could be used with different sensor integrated on underwear, It wouldn't be limited to one.

\section{RESULTS AND DISCUSSION}

Fig. 4 shows the capacitance values when the humidity was swept from $30 \%$ to $80 \%$ of relative humidity $(\mathrm{RH})$. Capacitance is directly affected by a RH change due to the change in permittivity of the substrate when absorb humidity. In the graph it is plotted the measurement results obtained for both, the external LCR meter and the charge/discharge method directly obtained with the microcontroller. It is observed that the capacitance value for $30 \% \mathrm{RH}$ is about $10 \mathrm{pF}$ and it reaches about $10 \mathrm{nF}$ for $80 \% \mathrm{RH}$. Despite of the values obtained for both measurement methods are slightly different, it does not have any impact on the system functionality because the system only needs to determinate if the system reaches a certain threshold value.

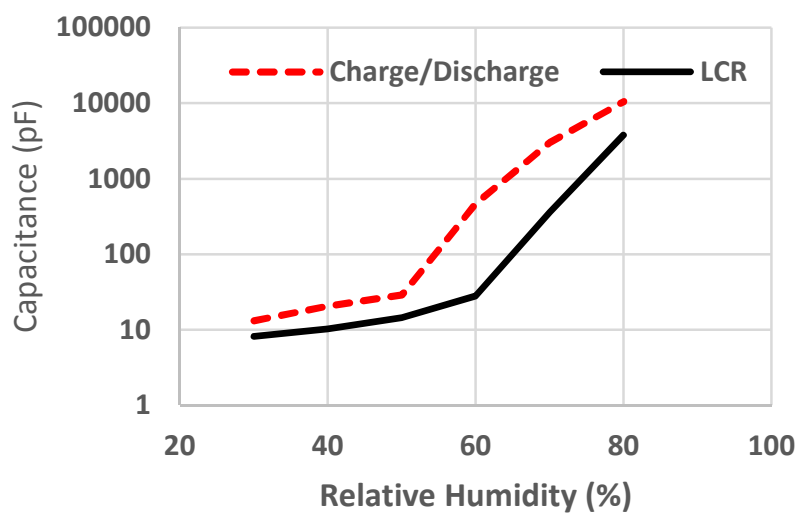

Fig. 4. Sensor capacitance measured with LCR meter and Charge/discharge method from $30 \% \mathrm{RH}$ to $80 \% \mathrm{RH}$.

A test with the diaper was performed to observe the sensor functionality in a real situation. The sensor was located under the diaper (Fig. 2) with the sensing area oriented along the diaper. To simulate urine, $100 \mathrm{ml}$ of water were used. The diaper chosen was supposed to absorb around $240 \mathrm{ml}$ of water without giving to the user wet feeling. The measurement results are shown in Fig. 5. After 600 seconds, $100 \mathrm{~mL}$ of water were poured into the diaper. The sensor capacitance increases instantly after poured the water due to the diaper abortion, which expels moisture from the inside of the diaper to the outside, providing a drying feeling for the user, the outer diaper starts to being wet because that flux of moisture. It is observed that in less than 120 seconds the capacitance value from the sensor change significantly, from $37 \mathrm{pF}$ to $149 \mu \mathrm{F}$, which is the higher point in the graph. Notice that before poured the water, the capacitance is $37 \mathrm{pF}$ which corresponds with an environmental humidity of about $55 \%$ according to sensor behavior measured with the charge/discharge method. When the diaper was removed to study the recovery time the sensor capacitance was reduced up to $126 \mathrm{pF}$ in 600 seconds. The sensor capacitance doesn't recover the original capacitance, due to hydrophilic behavior of the cotton. However, this point it is accepted as recovered, since it is possible to start another detection without expect false detections 


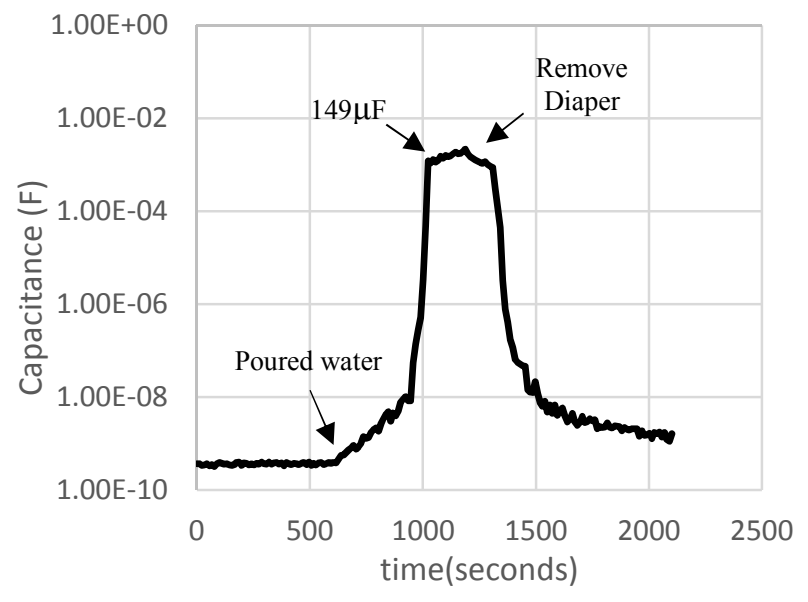

Fig. 5. Sensor capacitance evolution when $100 \mathrm{~mL}$ of water are poured on the diaper.

\section{CONCLUSIONS}

In this paper, a wearable system to detect urine leaks based on a textile sensor is presented. The system consists of a textile sensing part developed with a conductive yarn interdigital pattern embroidered on a standard underwear, an additional electronic for signal acquisition and a wireless transmission module. The system is able to detect urine leakage, even though the patient wears a diaper. The results demonstrated its functionally to develop a real time monitoring system to be used at retirement homes and/or medical facilities.

\section{ACKNOWLEDGMENT}

This work was supported by Spanish Government-MINECO under Project TEC2016-79465-R, AGAUR-UPC(2020 FI_B 00028) and Terrasa's city council "Beques de Recerca per a estudiants de centres universitars amb seu a Terrassa"

\section{References}

[1]. G. Matzeu, L. Florea, and D. Diamond, 'Advances in wearable chemical sensor design for monitoring biological fluids', Sensors Actuators B Chem., vol. 211, pp. 403-418, May 2015.

[2]. Yang, Shuozhi, and Qingguo Li. "Inertial sensor-based methods in walking speed estimation: a systematic review." Sensors (Basel, Switzerland) vol. 12,5 (2012): 6102-16. doi:10.3390/s120506102.

[3]. BR Greene, et al. "Longitudinal assessment of falls in patients with Parkinson's disease using inertial sensors and the Timed Up and Go test." Journal of rehabilitation and assistive technologies engineering vol. 5 2055668317750811. 12 Jan. 2018, poured

[4]. G. Tartare, X. Zeng and L. Koehl, "Development of a wearable system for monitoring the firefighter's physiological state," 2018 IEEE Industrial Cyber-Physical Systems (ICPS), St. Petersburg, 2018, pp. 561-566.

[5]. L. M. Castano and A. B. Flatau, 'Smart fabric sensors and e-textile technologies: A review', Smart Mater. Struct., vol. 23, no. 5, 2014.

[6]. J.-F. Wu et al., 'Human Limb Motion Detection with Novel Flexible Capacitive Angle Sensor Based on Conductive Textile', electronics, vol. 7, no. 192, 2018.

[7]. J. Deignan et al., "Textile piezoresistive sensors for on-body measurement of spinal extension," 2016 IEEE SENSORS, Orlando, FL, 2016, pp. 1-3.

[8]. T. Agcayazi, M. McKnight, et al., "A finger touch force detection method for textile based capacitive tactile sensor arrays," 2016 IEEE SENSORS, Orlando, FL, 2016, pp. 1-3.

[9]. M. Martínez-Estrada, B. Moradi, R. Fernández-Garcia, and I. Gil, 'Impact of Conductive Yarns on an Embroidery Textile Moisture Sensor', Sensors, vol. 19, no. 5, p. 1004, 2019.

[10]. M.I.Orna-Estebana et al. ' Prevalence of urinary incontinence in two urban health centres' SEMERGEN - Medicina de Familia, Volume 37, Issue 7, August-September 2011, Pages 347-351

[11]. M. Martinez-Estrada, B. Moradi, R. Fernández-Garcia, and I. Gil, 'Impact of manufacturing variability and washing on embroidery textile sensors', Sensors (Switzerland), vol. 18, no. 11, 2018.

[12]. Yao-Wen Chang et al., "Charge-based capacitance measurement for bias-dependent capacitance," in IEEE Electron Device Letters, vol. 27, no. 5, pp. 390-392, May 2006. 